BI-TP 99/03

\title{
Infrared behavior of the gluon propagator in lattice Landau gauge: the three-dimensional case
}

\author{
Attilio Cucchieri* \\ Fakultät für Physik, Universität Bielefeld, D-33615 Bielefeld, GERMANY
}

(October 13, 2018)

\begin{abstract}
We evaluate numerically the three-momentum-space gluon propagator in the lattice Landau gauge, for three-dimensional pure- $S U(2)$ lattice gauge theory with periodic boundary conditions. Simulations are done for nine different values of the coupling $\beta$, from $\beta=0$ (strong coupling) to $\beta=6.0$ (in the scaling region), and for lattice sizes up to $V=64^{3}$. In the limit of large lattice volume we observe, in all cases, a gluon propagator decreasing for momenta smaller than a constant value $p_{d e c}$. From our data we estimate $p_{d e c} \approx 350 \mathrm{MeV}$. The result of a gluon propagator decreasing in the infrared limit has a straightforward interpretation as resulting from the proximity of the so-called first Gribov horizon in the infrared directions.
\end{abstract}

\section{INTRODUCTION}

The infrared behavior of the gluon propagator in lattice Landau gauge has been the subject of several numerical studies [1 10]. In fact, although this propagator is a non-gauge-invariant quantity, the study of its infrared behavior provides a powerful tool for increasing our understanding of QCD, and for gaining insight into the physics of confinement in non-abelian gauge theories (see for example [11]). In particular, the infrared behavior of the gluon propagator can be directly related [12] to the behavior of the Wilson loop at large separations, and to the existence of an area law.

On the lattice, the Landau gauge condition is imposed [1, 13] by finding a gauge transformation which brings the functional $\mathcal{E}_{U}[g]$, defined in eq. (4) below, to a minimum. A lattice configuration satisfying this minimizing condition belongs to the region $\Omega$ of transverse configurations, for which the Faddeev-Popov operator is nonnegative [14 16]. This region is delimited by the so-called first Gribov horizon, defined as the set of configurations for which the smallest non-trivial eigenvalue of the Faddeev-Popov operator is zero. (The Faddeev-Popov operator has a trivial null eigenvalue, corresponding to a constant eigenvector.)

The restriction of the configuration space to the region $\Omega$ implies a rigorous inequality [14 [16] for the Fourier components of the gluon field $A_{\mu}(x)$. From this inequality, which is a consequence of the positiveness of the FaddeevPopov operator only, it follows that the region $\Omega$ is bounded by a certain ellipsoid $\Theta$. This bound implies proximity of the first Gribov horizon in infrared directions, and consequent suppression of the low-momentum components of the gauge field, a result already noted by Gribov in Reference [17. This bound also causes a strong suppression of the gluon propagator in the infrared limit (i.e. for momentum $p \rightarrow 0$ ). In fact, Zwanziger proved [16, 18] that, in four dimensions and in the infinite-volume limit, the gluon propagator is less singular than $p^{-2}$ in the infrared limit and that, very likely, it does vanish in this limit. More precisely, in Reference [18] it was proven that, in the infinite-volume limit, the "gluon propagator" $D(H, p)$ goes to zero as $p \rightarrow 0$ for almost every $H$. Here $H$ should be interpreted as the strength of a spatially-modulated magnetic field coupled to the gluon field $A_{\mu}(x)$, and the standard gluon propagator is obtained for $H=0$. A similar result holds in three dimensions: one obtains 16,18 that, in the infinite-volume limit, the gluon propagator must be less singular than $p^{-1}$ as $p \rightarrow 0$ and that, very likely, it vanishes in the infrared limit.

${ }^{*}$ E-mail address: attilio@physik.uni-bielefeld.de. 
A gluon propagator vanishing in the infrared limit was also found - in four dimensions - by Gribov [17]. More precisely, he obtained the expression $p^{2} /\left(p^{4}+\gamma\right)$, where the mass scale $\gamma^{1 / 4}$ arises when the configuration space is restricted to the region $\Omega$. This propagator agrees with the zeroth-order perturbative prediction $p^{-2}$ at large momenta, but gives a null propagator at $p=0$. The mass scale $\gamma^{1 / 4}$ marks the transition point between the perturbative and the nonperturbative regimes. A propagator that is a generalization of the one obtained by Gribov has also been introduced in Reference [19] as an Ansatz for a non-perturbative solution of the gluon Dyson-Schwinger equation (DSE).

Let us notice that a gluon propagator vanishing in the infrared limit is in complete contradiction with the $p^{-4}$ singularity obtained when the gluon DSE is approximately solved in the infrared limit [11,20]. However, a recent study [21] has shown that this singularity is obtained only if the ghost contributions to the gluon DSE are neglected. In fact, when these contributions are included, the gluon propagator vanishes in the infrared limit [21], in qualitative agreement with References [16 18$]$.

In this paper we present the first numerical study of the infrared behavior of the gluon propagator in three dimensions.1 As explained in References [23 25], nonabelian gauge theories in three dimensions are similar to their four-dimensional counterparts, and results obtained in the three-dimensional case can teach us something about the more realistic four-dimensional theories. Of course, the advantage of using a three-dimensional lattice is the possibility of simulating lattice sizes larger than those used in the four-dimensional case. This is particularly important in the study of the gluon propagator since Zwanziger's prediction of an infrared-suppressed gluon propagator is valid only in the infinite-volume limit.

We recall that in some recent numerical studies (in four dimensions) [5.6] a sensible change in the infrared behavior of the gluon propagator has been observed for momenta smaller than a turn-over value $p_{t o}$, in agreement with the prediction of a gluon propagator less singular than $p^{-2}$ in the infrared limit. Also, the numerical data obtained in Reference [3] have been successfully fitted by a Gribov-like formula [4]. Finally, in References [7] 9] we have observed - for the $S U(2)$ group, in four dimensions, in the strong-coupling regime and in the limit of large lattice volume - a gluon propagator decreasing as the momentum goes to zero. A similar result has also been obtained recently 10] for the $S U(3)$ group in the strong-coupling regime, both in the quenched and in the unquenched case. Let us notice that Zwanziger's predictions [16,18] for the gluon propagator are $\beta$-independent: in fact, they are derived only from the positiveness of the Faddeev-Popov operator when the lattice Landau gauge is imposed. Thus, results in the strong-coupling regime (i.e. $\beta \rightarrow 0$ ) are a valid test of these predictions. Nevertheless, it is important to extend this result to higher values of $\beta$, possibly up to the scaling region. Of course, as $\beta$ increases, one needs to consider larger lattice sizes in order to probe the infrared behavior of the gluon propagator. Moreover, as said above, the prediction we want to test applies only in the infinite-volume limit. In four dimensions it was found [7] that the lattice size at which an infrared-decreasing gluon propagator starts to be observed increases with the coupling. This makes practically unfeasible, with present computational resources, to study numerically the infrared behavior of the gluon propagator in the four-dimensional case and at values of $\beta$ in the scaling region. Our hope is that in this work, by studying the three-dimensional case, we can consider lattice volumes that are large enough to allow a decreasing gluon propagator to be observed not only in the strong-coupling regime but also in the scaling region.

\section{DEFINITIONS AND NOTATIONS}

We consider a standard Wilson action for $S U(2)$ lattice gauge theory in 3 dimensions

$$
S[U] \equiv \frac{4}{a g^{2}} \frac{1}{2} \sum_{\mu, \nu=1}^{3} \sum_{x}\left\{1-\frac{\operatorname{Tr}}{2}\left[U_{\mu}(x) U_{\nu}\left(x+e_{\mu}\right) U_{\mu}^{-1}\left(x+e_{\nu}\right) U_{\nu}^{-1}(x)\right]\right\}
$$

where $U_{\mu}(x) \in S U(2)$ are link variables, $g$ is the bare coupling constant, $a$ is the lattice spacing, and $e_{\mu}$ is a unit vector in the positive $\mu$ direction. We assume periodic boundary conditions. For the matrices $U \in S U(2)$ we use the parametrization $U \equiv U_{0} \mathbb{1}+i \vec{U} \cdot \vec{\sigma}$, where $\mathbb{1}$ is the $2 \times 2$ identity matrix, the components of $\vec{\sigma} \equiv\left(\sigma^{1}, \sigma^{2}, \sigma^{3}\right)$ are the Pauli matrices, $U_{0} \in \Re, \vec{U} \in \Re^{3}$ and $U_{0}^{2}+\vec{U} \cdot \vec{U}=1$. Notice that in eq. (1) the lattice spacing $a$ is necessary in order to make the action $S[U]$ dimensionless; in fact, in the three-dimensional case [23 25], the coupling $g^{2}$ has dimension of mass, and in order to obtain a dimensionless lattice coupling we have to set $\beta \equiv 4 /\left(a g^{2}\right)$.

${ }^{1}$ Preliminary results have been reported in [22. 
We define the gauge field $A_{\mu}(x)$, which belongs to the $\mathcal{S U}(2)$ Lie algebra, as

$$
A_{\mu}(x) \equiv \frac{1}{2}\left[U_{\mu}(x)-U_{\mu}^{\dagger}(x)\right]=i \vec{U}_{\mu}(x) \cdot \vec{\sigma} .
$$

We also define

$$
A_{\mu}^{b}(x) \equiv \frac{\operatorname{Tr}}{2 i}\left[A_{\mu}(x) \sigma^{b}\right]=U_{\mu}^{b}(x),
$$

where $\sigma^{b}$ is a Pauli matrix. Note that $a^{-1} A_{\mu}^{b}(x)$ approaches $(1 / 2) g\left[A^{(\text {cont })}\right]_{\mu}^{b}(x)$ in the continuum limit, where $\left[A^{(\text {cont })}\right]_{\mu}^{b}(x)$ is the (unrenormalized) conventional vector potential.

In order to fix the lattice Landau gauge we look for a local minimum² of the functional [1, 13]

$$
\mathcal{E}_{U}[g] \equiv 1-\frac{1}{3 V} \sum_{\mu=1}^{3} \sum_{x} \frac{\operatorname{Tr}}{2}\left[g(x) U_{\mu}(x) g^{\dagger}\left(x+e_{\mu}\right)\right],
$$

where $g(x) \in S U(2)$ are site variables, and $V \equiv N_{s}^{2} N_{t}$ is the lattice volume. (Here $N_{s}$ is the number of lattice sites in the two spatial directions, and $N_{t}$ is the number of lattice sites in the time direction.) If the configuration $\left\{U_{\mu}(x)\right\}$ is a stationary point of the functional $\mathcal{E}_{U}[g]$ then [13] the lattice divergence of $A_{\mu}^{b}(x)$ is null, namely

$$
(\nabla \cdot A)^{b}(x) \equiv \sum_{\mu=1}^{3}\left[A_{\mu}^{b}(x)-A_{\mu}^{b}\left(x-e_{\mu}\right)\right]=0 \quad \forall x, b .
$$

This is the lattice formulation of the usual (continuum) Landau gauge-fixing condition. By summing eq. (5) over the components $x_{\mu}$ of $x$ with $\mu \neq \nu$, for fixed $\nu$, and using the periodicity of the lattice, it is easy to check [1] that if the Landau gauge-fixing condition is satisfied then the quantities

$$
Q_{\nu}^{b}\left(x_{\nu}\right) \equiv \sum_{\mu \neq \nu} \sum_{x_{\mu}} A_{\nu}^{b}(x)
$$

are constant, i.e. independent of $x_{\nu}$.

\section{GLUON PROPAGATOR ON THE LATTICE}

The lattice space-time gluon propagator is given by

$$
D_{\mu \nu}^{b c}(x-y) \equiv\left\langle A_{\mu}^{b}(x) A_{\nu}^{c}(y)\right\rangle .
$$

To go to momentum space we can use Formula (3.1a) in Reference 16] and obtain

$$
\begin{aligned}
& D(0) \equiv \frac{1}{9 V} \sum_{\mu, b}\left\langle\left[\sum_{x} A_{\mu}^{b}(x)\right]^{2}\right\rangle \\
& D(k) \equiv \frac{1}{6 V} \sum_{\mu, b}\left\langle\left\{\left[\sum_{x} A_{\mu}^{b}(x) \cos (2 \pi k \cdot x)\right]^{2}+\left[\sum_{x} A_{\mu}^{b}(x) \sin (2 \pi k \cdot x)\right]^{2}\right\}\right\rangle .
\end{aligned}
$$

Here $\mu$ goes from 1 to 3 , and $k$ has components $\left(k_{x}, k_{y}, k_{t}\right)$. In our simulations we consider the values $k_{x} N_{s}=k_{y} N_{s}=$ 0,1 and $k_{t} N_{t} \equiv 0,1, \ldots, N_{t}-1$; and the momentum-space gluon propagator is studied as a function of the magnitude of the lattice momentum

\footnotetext{
${ }^{2}$ Here we do not consider the problem of searching for the absolute minimum of the functional $\mathcal{E}_{U}[g]$, which defines the so-called minimal Landau gauge [18]. In fact, as stressed in the Introduction, the prediction of an infrared-suppressed gluon propagator is valid for any configuration in the region $\Omega$, i.e. for local as well absolute minima of the functional $\mathcal{E}_{U}[g]$.
} 


$$
p(k) \equiv \sqrt{\sum_{\mu=1}^{3} p_{\mu}^{2}(k)} \equiv 2 \sqrt{\sum_{\mu=1}^{3} \sin ^{2}\left(\pi k_{\mu}\right)} .
$$

If we define the momentum-space gluon field as

$$
\widetilde{A}_{\mu}^{b}(k) \equiv \sum_{x} A_{\mu}^{b}(x) \exp \left[2 \pi i\left(k \cdot x+k_{\mu} / 2\right)\right]
$$

then eqs. (8) and (9) can be rewritten as

$$
\begin{aligned}
& D(0) \equiv \frac{1}{9 V} \sum_{\mu, b}\left\langle\left[\widetilde{A}_{\mu}^{b}(0)\right]^{2}\right\rangle \\
& D(k) \equiv \frac{1}{6 V} \sum_{\mu, b}\left\langle\widetilde{A}_{\mu}^{b}(k) \widetilde{A}_{\mu}^{b}(-k)\right\rangle .
\end{aligned}
$$

Notice that $D(0)$ in eq. (8) [or in eq. (12)] is not given by $D(k)$ in eq. (9) [or in eq. (13)] at $k=0$. The difference is due to the Landau gauge condition - the continuum-like condition as in eq. (5) - which in momentum space reads

$$
\sum_{\mu=1}^{3} p_{\mu}(k) \widetilde{A}_{\mu}^{b}(k)=0 \quad \forall k, b .
$$

If $k \neq(0,0,0)$ we obtain that only two of the three Lorentz components of $\widetilde{A}^{b}(k)$ - and therefore of $A^{b}(x)-$ are independent. This explains the factor 6 (instead of 9) in eqs. (9) and (13).

Let us also note that the zero three-momentum gluon propagator $D(0)$ can be written as

$$
D(0)=\frac{V}{9} \sum_{\mu, b}\left\langle\left(\mathcal{A}_{\mu}^{b}\right)^{2}\right\rangle,
$$

where $\mathcal{A}_{\mu}^{b} \equiv V^{-1} \sum_{x} A_{\mu}^{b}(x)$ is the zero-momentum component of the gluon field $A_{\mu}^{b}(x)$. Notice that a nonzero value for the constants $\mathcal{A}_{\mu}^{b}$ is a lattice artifact related to the use of periodic boundary conditions and to the finiteness of the volume. In fact, after the Landau gauge condition is imposed, these constants are identically null — even on a finite lattice - if free boundary conditions are considered [26], while in the periodic case they must go to zero in the infinite-volume limit [15,16]. Of course, due to the volume factor in eq. (15), the latter result does not imply that $D(0)$ should be zero in the infinite-volume limit. Nevertheless, as mentioned in the Introduction, it has been proven by Zwanziger 16.18] that, in this limit and in three dimensions, the gluon propagator is less singular at momentum $p=0$ than $p^{-1}$ and that, very likely, it vanishes in the infrared limit.

\section{NUMERICAL SIMULATIONS}

In Table 1 we report, for each pair $(\beta, V)$, the parameters used for the simulations. ${ }^{3}$ Overall, we have considered about 4100 configurations. In all our runs we have started from a randomly chosen lattice gauge configuration. To thermalize the gauge configuration $\left\{U_{\mu}(x)\right\}$ we use a hybrid overrelaxed (HOR) algorithm [25]27, i.e. $m$ microcanonical (or energy-conserving) update sweeps are done, followed by one standard local ergodic update (heat-bath sweep) of the lattice. In order to optimize the efficiency of the heat-bath code, we implement two different $S U(2)$ generators (methods 1 and 2 described in Appendix A of Reference [28], with $h_{\text {cutoff }}=2$ ). In our case we did not try to find the best tuning for the value of $m$. By analogy with the four-dimensional case [7], 8] we set $m=N_{s} / 2$.

For all the pairs $(\beta, V)$, we evaluated the integrated autocorrelation tim 1 f $\tau_{\text {int }}$ for the Wilson loops

\footnotetext{
${ }^{3}$ Computations were performed on a SUN Ultra-1 and on a SUN Ultra-2 at the Università di Roma "Tor Vergata", where part of this work has been done, and on an ALPHAstation 255 at the ZiF-Center in Bielefeld.

${ }^{4}$ For a definition see for example [29]. To evaluate the integrated autocorrelation time we use an automatic windowing procedure 29] with two different window factors (6 and 15). We also employ a method 30] based on a comparison between the naive statistical error with a jack-knife binning error [13]. In all cases we checked that these three estimates are in agreement.
} 


$$
W_{l, l} \equiv \frac{1}{3 V} \frac{\operatorname{Tr}}{2} \sum_{\nu>\mu} \sum_{x} U_{\mu, \nu}^{l, l}(x) \quad l=1,2,4, \ldots, N_{s} / 2
$$

where

$$
\begin{aligned}
U_{\mu, \nu}^{l, l}(x) \equiv & U_{\mu}(x) \ldots U_{\mu}\left(x+(l-1) e_{\mu}\right) U_{\nu}\left(x+(l-1) e_{\mu}\right) \ldots U_{\nu}\left(x+(l-1) e_{\mu}+(l-1) e_{\nu}\right) \\
& \times U_{\mu}^{-1}\left(x+(l-1) e_{\mu}+(l-1) e_{\nu}\right) \ldots U_{\mu}^{-1}\left(x+(l-1) e_{\nu}\right) U_{\nu}^{-1}\left(x+(l-1) e_{\nu}\right) \ldots U_{\nu}^{-1}(x),
\end{aligned}
$$

and for the Polyakov loops

$$
P_{\mu} \equiv \frac{N_{\mu}}{V} \sum_{\nu \neq \mu} \sum_{x_{\nu}} \frac{\operatorname{Tr}}{2} \prod_{n_{\mu}=1}^{N_{\mu}} U_{\mu}\left(x+n_{\mu} e_{\mu}\right) \quad \mu=1,2,3 .
$$

In all cases we obtained $\tau_{\text {int }} \lesssim 1$. (Note that $\tau_{\text {int }}=0.5$ indicates that two successive configurations generated in the Monte Carlo simulation are independent.) Since, for all pairs $(\beta, V)$ and for all quantities, the number of sweeps between two consecutive configurations used for evaluating the gluon propagator (see Table I]) is much larger than the corresponding integrated autocorrelation time, we may conclude that these configurations are essentially statistically independent.

For the numerical gauge fixing we use the so-called stochastic overrelaxation algorithm [31, 32]. In all our simulations we stop the gauge fixing when the condition

$$
\frac{1}{V} \sum_{x, b}\left[(\nabla \cdot A)^{b}(x)\right]^{2} \leq 10^{-12}
$$

is satisfied. [See eq. (5) for the definition of the lattice divergence $(\nabla \cdot A)^{b}(x)$ of the gluon field $A_{\mu}^{b}(x)$.] This is equivalent [32] to fixing the minimizing functional $\mathcal{E}_{U}[g]$ up to about one part in $10^{12}$. In the final gauge-fixed configuration we also evaluate [32]

$$
Q \equiv \frac{1}{9} \sum_{\nu} \frac{1}{N_{\nu}} \sum_{x_{\nu}, b}\left[Q_{\nu}^{b}\left(x_{\nu}\right)-\widehat{Q}_{\nu}^{b}\right]^{2}\left[\widehat{Q}_{\nu}^{b}\right]^{-2}
$$

where $\widehat{Q}_{\nu}^{b} \equiv N_{\nu}^{-1} \sum_{x_{\nu}} Q_{\nu}^{b}\left(x_{\nu}\right)$, and $Q_{\nu}^{b}\left(x_{\nu}\right)$ has been defined in eq. (6). The quantity $Q$ should be zero when the configuration is gauge-fixed, and it is a good estimator of the quality of the gauge fixing. As in References [32, we found that the stochastic overrelaxation algorithm is very efficient in fighting critical slowing-down 29], and in making the quantities $Q_{\nu}^{b}\left(x_{\nu}\right)$ constant, i.e. $Q \approx 0$. In particular, by averaging over all the gauge-fixed configurations, we find $Q=3.5(10) \times 10^{-6}$. We also obtain $Q \leq 10^{-8}$ for $69 \%$ of the gauge-fixed configurations.

\section{A. String tension and lattice spacing}

For each coupling $\beta$ we evaluate the average plaquette $\left\langle W_{1,1}\right\rangle$ (see Table [1]). Results for $\beta=5.0$ and 6.0 are in agreement with the data reported in Table 15 of Reference [25]. In Figure 11 we also plot $\left\langle W_{1,1}\right\rangle$ as a function of the coupling $\beta$, and we compare the numerical data with the leading strong-coupling expansion $\beta / 4$ and weak-coupling expansion $\exp (-1 / \beta)$. It is clear that the crossover region from strong coupling to weak coupling occurs around $\beta \approx 3$, in agreement with Reference [23], and that our simulations range from the strong-coupling region up to the weak-coupling one.

Following Reference [25] we also evaluate, for $\beta \geq 3.4$, the tadpole-improved coupling $\beta_{I} \equiv \beta\left\langle W_{1,1}\right\rangle$ (see Table [1]). Then, by using the fit given in eq. (67) of that reference (which is valid for $\beta \gtrsim 3.0$ ), we calculate the string tension $\sqrt{\sigma}$ in lattice units, and the inverse lattice spacing $a^{-1}$ using the input value $\sqrt{\sigma}=0.44 \mathrm{GeV}$ (see the last two columns in Table [I]).

Finally, in Table III we report (for each $\beta \geq 3.4$ ) the lattice spacing in $\mathrm{fm}$, the largest lattice volume $V_{\max }$ considered, the corresponding physical volume in $\mathrm{fm}^{3}$, and the smallest non-zero momentum (in $\mathrm{GeV}$ ) that can be considered for that lattice. Thus, in this work, we can explore the infrared behavior of the gluon propagator for momenta as small as $p \approx 110 \mathrm{MeV}$, in relatively large physical volumes, and for couplings $\beta$ above the strong-coupling region. Let us notice that, if we compare the data for the string tension (in lattice units) with data obtained for the $S U(2)$ group in four dimensions (see for example Table 3 in Reference [33]), then our largest value of $\beta$, namely 6.0, corresponds to $\beta \approx 2.4$ in the four dimensional case. 


\section{B. Gribov copies}

In this work we do not consider the problem of Gribov copies (see for example [7] 8 and references therein.) This is motivated by our finding in the study of the four-dimensional case. In fact, in References [7],8] we checked that, for the $S U(2)$ group in the four-dimensional case, the influence of Gribov copies on the gluon propagator is of the order of magnitude of the numerical accuracy. (A similar result has also been obtained for the Coulomb gauge [34].) In fact, from Table 2 in Reference [8], it is clear that data corresponding to the minimal Landau gauge (absolute minima of the functional $\mathcal{E}_{U}[g]$ ) are in complete agreement, within statistical errors, with those obtained in a generic Landau gauge (local minima of $\mathcal{E}_{U}[g]$ ). In particular, this seems to be the case even at small values of the coupling $\beta$, namely in the strong-coupling regime, where the number of Gribov copies is higher and their effects, if present, should be larger and more easily detectable.

\section{C. $Z(2)$ symmetry}

In References [35] it was shown for the four-dimensional case that, at very large $\beta$, the data for the gluon propagator are strongly affected by the broken $Z(2)$ symmetry. In particular, one can consider all the possible combinations of signs of the average Polyakov loops $\left\langle P_{\mu}\right\rangle$ [see eq. (18)], for a total of $2^{4}=16$ different states. Then, if the expectation values are evaluated only over configurations belonging to the same state, the gluon propagator takes different values in different states [35]. Here we did the same analysis at $\beta=5.0$ and lattice volume $V=16^{3}$ with 1000 configurations. Since we work in three dimensions, there are $2^{3}=8$ possible states, i.e. combinations of the signs of the average Polyakov loops. We obtain that, also in this case, the gluon propagator depends strongly on the state used for evaluating the expectation value. For example, for $k=(0,0,1)$ the smallest value $-D(k)=1.50(3)$ - is obtained for the state characterized by positive Polyakov loops in the three directions, while the largest value $-D(k)=1.68(3)-$ corresponds to the state characterized by negative Polyakov loops in the three directions. The two values clearly differ by several standard deviations. A similar result is obtained when other momenta $k$ are considered. This observation may explain why the data for the gluon propagator are usually characterized by large statistical fluctuations.

\section{INFRARED BEHAVIOR OF THE GLUON PROPAGATOR}

In Figures 2 we plot the data for the gluon propagator [see eqs. (8) and (9)] as a function of the lattice momentum $p(k)$, defined in eq. (10), for different lattice volumes $V$ and couplings $\beta$. Our data confirm previous results [7 10] obtained in the strong-coupling regime for the four-dimensional case: the gluon propagator is decreasing as $p(k)$ decreases, provided that $p(k)$ is smaller than a value $p_{d e c}$. Also, as in four dimensions, the lattice size at which this behavior for the gluon propagator starts to be observed increases with the coupling $\beta$. In particular, in the strongcoupling regime, this propagator is clearly decreasing as $p(k)$ goes to zero, even for relatively small lattice volumes (see Figures 2 and 3). On the contrary, for $\beta \geq 3.4$ (see Figure 1 ), this propagator is increasing (monotonically) in the infrared limit for $V=16^{3}$, while it is decreasing at the largest lattice volume considered.

Let us also notice that, at high momenta, there are very small finite-size effects, at all values of $\beta$. The situation is completely different in the small-momenta sector, as already stressed above. Moreover, the value $D(0)$ of the gluon propagator at zero momentum decreases monotonically as the lattice volume increases (see for example the case $\beta=5.0$ in Figure 4 ). These results suggest a finite value for $D(0)$ in the infinite-volume limit, but it is not clear whether this value would be zero or a strictly positive constant. Therefore, the possibility of a zero value for $D(0)$ in the infinite-volume limit is not ruled out.

Finally, in Figure 司 we plot (for $\beta=5.0$ and 6.0 and $V=64^{3}$ ) the data for the gluon propagator $D(k)$ with the choice $k=\left(0,0, k_{t}\right)$, together with the data for $k=\left(1,1, k_{t}\right)$. In both cases the two sets of data seem to fall on a single curve, i.e. we see no sign of breaking of rotational invariance.

As said in Section II, with our definition of the gluon field [see eqs. (21) and (3)] the quantity $a^{-1} A_{\mu}^{b}(x)$ approaches $(1 / 2) g\left[A^{(\text {cont })}\right]_{\mu}^{b}(x)$ in the continuum limit $a \rightarrow 0$, where $\left[A^{(\text {cont })}\right]_{\mu}^{b}(x)$ is the (unrenormalized) conventional vector potential. In the same limit, a $D(k)$ approaches $g^{2} D^{(\text {cont })}(k) / 4$, where $D^{(\text {cont })}(k)$ is the unrenormalized continuum gluon propagator. [We recall that, in three dimensions, $D^{(\text {cont })}(k)$ has mass dimension -2 and $g^{2}$ has mass dimension

\footnotetext{
${ }^{5}$ In particular see Figure 1 in Reference 9.
} 
1.] In Figure 6 we plot $a D(k)$ (in $\mathrm{GeV}^{-1}$ ) as a function of the momenta $a^{-1} p(k)$ (in $\mathrm{GeV}$ ) for three different values of the coupling $\beta$ : 3.4,4.2 and 5.0. In all cases we consider the largest lattice volume available (see Table Ii). The data show good scaling in the region where finite-size effects are negligible, i.e. in the limit of large momenta (see Figure 6). 6 On the contrary, the scaling is poorer in the infrared limit as expected. Nevertheless, we can see that the gluon propagator is decreasing for momenta $p \lesssim p_{d e c}$, and that the value of $p_{d e c}$ (in physical units) is practically $\beta$-independent. From our data (see Figure $6 \mathrm{~b}$ ) we can set $p_{d e c} \approx 350 \mathrm{MeV}$. Let us notice that $p_{\text {dec }}$ corresponds to the mass scale $\gamma^{1 / 4}$ in a Gribov-like propagator.

Finally, for the same set of data we consider (see Figure 7) the plot of the dimensionless product $p(k) D(k)$ as a function of $a^{-1} p(k)$. Since $p(k) D(k)$ is decreasing for $a^{-1} p(k) \lesssim 700 \mathrm{MeV}$, we can say that the gluon propagator is less singular than $p^{-1}$ in the infrared limit, in agreement with Zwanziger's prediction. We notice that the turn-over value $p_{t o} \approx 700 \mathrm{MeV}$ is in good agreement with the result obtained recently in four dimensions for the $S U(3)$ group (see Figure 8 in Reference [6]).

\section{CONCLUSIONS}

We think that our data for the gluon propagator are very interesting. The prediction [16 19,21] of a propagator decreasing for momenta $p \lesssim p_{d e c}$ is clearly verified numerically for several values of the coupling $\beta$, ranging from the strong-coupling regime to the scaling region. Moreover, as in the four-dimensional case [7 9], it appears that the lattice size at which this behavior for the gluon propagator starts to be observed increases with the coupling. This requirement of large lattice volumes could explain why a decreasing gluon propagator has never been observed in the scaling region for the four-dimensional case [1] 10 .

As mentioned above, our data in the strong-coupling regime and in three dimensions are in qualitative agreement with results obtained at small $\beta$ in the four dimensional case $[7[10]$. This strongly suggests to us that a similar analogy will hold — in the limit of large lattice volumes — for couplings $\beta$ in the scaling region, leading to an infrared-suppressed gluon propagator also in the four-dimensional case.

\section{ACKNOWLEDGMENTS}

I would like to thank T. Mendes and D. Zwanziger for valuable discussions and suggestions. I also thank R. Alkofer and A. Hauck for e-mail correspondence. This work was partially supported by the TMR network Finite Temperature Phase Transitions in Particle Physics, EU contract no.: ERBFMRX-CT97-0122.

[1] J. E. Mandula and M. Ogilvie, Phys. Lett. B185 (1987) 127.

[2] R. Gupta et al., Phys. Rev. D36 (1987) 2813; P. Marenzoni et al., Phys. Lett. B318 (1993) 511; C. Bernard, C. Parrinello and A. Soni, Phys. Rev. D49 (1994) 1585; P. Marenzoni, G. Martinelli and N. Stella, Nucl. Phys. B455 (1995) 339.

[3] A. Nakamura et al., Gluon propagators and confinement, hep-lat/9506024, published in the Proceedings of the International RCNP Workshop on Color Confinement and Hadrons, Osaka (Japan) 22-24 March 1995.

[4] H. Aiso et al., Nucl. Phys. B (Proc. Suppl.) 53 (1997) 570.

[5] F. Gutbrod, A study of the gluon propagator in SU(2) lattice gauge theory, DESY-96-252 preprint; D. B. Leinweber et al., The structure of the gluon propagator, hep-lat/9809030; Modeling the gluon propagator, hep-lat/9809031; Asymptotic scaling and infrared behavior of the gluon propagator, hep-lat/9811027; D. B. Leinweber, J. I. Skullerud and A. G. Williams, The infrared behavior of the gluon propagator from lattice $Q C D$, hep-lat/9808033; Lattice gauge theory studies of the gluon propagator, hep-lat/9808037.

[6] D. B. Leinweber et al., Phys. Rev. D58 (1998) 031501.

[7] A. Cucchieri, Numerical results in minimal lattice Coulomb and Landau gauges: color-Coulomb potential and gluon and ghost propagators, PhD thesis, New York University (May 1996), UMI-97-02010 microfiche.

\footnotetext{
${ }^{6}$ We have checked scaling, in the limit of large momenta, also for the data at $\beta=6.0$. However, these data are not included in Figure 6 for clarity.
} 
[8] A. Cucchieri, Nucl. Phys. B508 (1997) 353.

[9] A. Cucchieri, Phys. Lett. B422 (1998) 233.

[10] H. Nakajima and S. Furui, Gluon propagator in the Landau gauge fixed lattice QCD simulation, hep-lat/9809078; The Landau gauge lattice QCD simulation and the gluon propagator, hep-lat/9809081.

[11] C. D. Roberts, Nonperturbative QCD with modern tools, nucl-th/9807026; F. T. Hawes, P. Maris and C. D. Roberts, Infrared behavior of propagators and vertices, nucl-th/9807056.

[12] B. West, Phys. Lett. B115 (1982) 468.

[13] K. G. Wilson, Recent developments in gauge theories, Proc. NATO Advanced Study Institute (Cargése, 1979), eds. G. 't Hooft et al. (Plenum Press, New York-London, 1980).

[14] G. Dell'Antonio and D. Zwanziger, Nucl. Phys. B326 (1989) 333.

[15] D. Zwanziger, Phys. Lett. B257 (1991) 168.

[16] D. Zwanziger, Nucl. Phys. B364 (1991) 127.

[17] V. N. Gribov, Nucl. Phys. B139 (1978) 1.

[18] D. Zwanziger, Nucl. Phys. B412 (1994) 657.

[19] M. Stingl, Phys. Rev. D34 (1986) 3863; Z. Phys. A353 (1996) 423.

[20] S. Mandelstam, Phys. Rev. D20 (1979) 3223; N. Brown and M. R. Pennington, Phys. Rev. D38 (1988) 2266; K. Buttner and M. R. Pennington, Phys. Rev. D52 (1995) 5220.

[21] L. von Smekal, R. Alkofer and A. Hauck, Phys. Rev. Lett. 79 (1997) 3591; Ann. Phys. 267 (1998) 1.

[22] A. Cucchieri and T. Mendes, Nucl. Phys. B (Proc. Suppl.) 63 (1998) 841; A. Cucchieri, Numerical study of the gluon propagator in lattice Landau gauge: the three-dimensional case, hep-lat/9810022.

[23] J. Ambjørn, J. G. Hey and S. Otto, Nucl. Phys. B210 (1982) 347.

[24] J. Ambjørn, C. Olesen and C. Peterson, Nucl. Phys. B244 (1984) 262; A. Irbäck and C. Peterson, Phys. Lett. B174 (1986) 99; M. Teper, Phys. Lett. B289 (1992) 115; Phys. Lett. B311 (1993) 223; H. D. Trottier, Improvement, dynamical fermions, and heavy quark screening in $Q C D_{3}$, hep-lat/9809183.

[25] M. Teper, $S U(N)$ gauge theories in (2+1)-dimensions, hep-lat/9804008.

[26] M. Schaden and D. Zwanziger, Horizon condition holds pointwise on finite lattice with free boundary conditions, hepth/9410019, published in the Proceedings of the Workshop on Quantum Infrared Physics, Paris (France) 6-10 June 1994.

[27] F. R. Brown and T. J. Woch, Phys. Rev. Lett. 58 (1987) 2394; S. L. Adler, Nucl. Phys. B (Proc. Suppl.) 9 (1989) 437; K. M. Decker and Ph. de Forcrand, Nucl. Phys. B (Proc. Suppl.) 17 (1990) 567; U. Wolff, Nucl. Phys. B (Proc. Suppl.) 17 (1990) 93; Phys. Lett. B288 (1992) 166; M. Lüscher et al., Nucl. Phys. B389 (1993) 247.

[28] R. G. Edwards et al., Nucl. Phys. B380 (1992) 621.

[29] A. D. Sokal, Monte Carlo methods in statistical mechanics: foundations and new algorithms, Cours de Troisième Cycle de la Physique en Suisse Romande, Lausanne (Suisse) June 1989.

[30] G. de Divitiis et al., Nucl. Phys. B437 (1995) 447.

[31] Ph. de Forcrand and R. Gupta, Nucl. Phys. B (Proc. Suppl.) 9 (1989) 516.

[32] A. Cucchieri and T. Mendes, Nucl. Phys. B471 (1996) 263; Nucl. Phys. B (Proc. Suppl.) 53 (1997) 811; and in preparation.

[33] J. Fingberg, U. Heller and F. Karsch, Nucl. Phys. B392 (1993) 493.

[34] A. Cucchieri and D. Zwanziger, in preparation.

[35] G. Damm, W. Kerler and V. K. Mitrjushkin, Nucl. Phys. (Proc. Suppl.) 63 (1998) 251; Phys. Lett. B433 (1998) 88. 


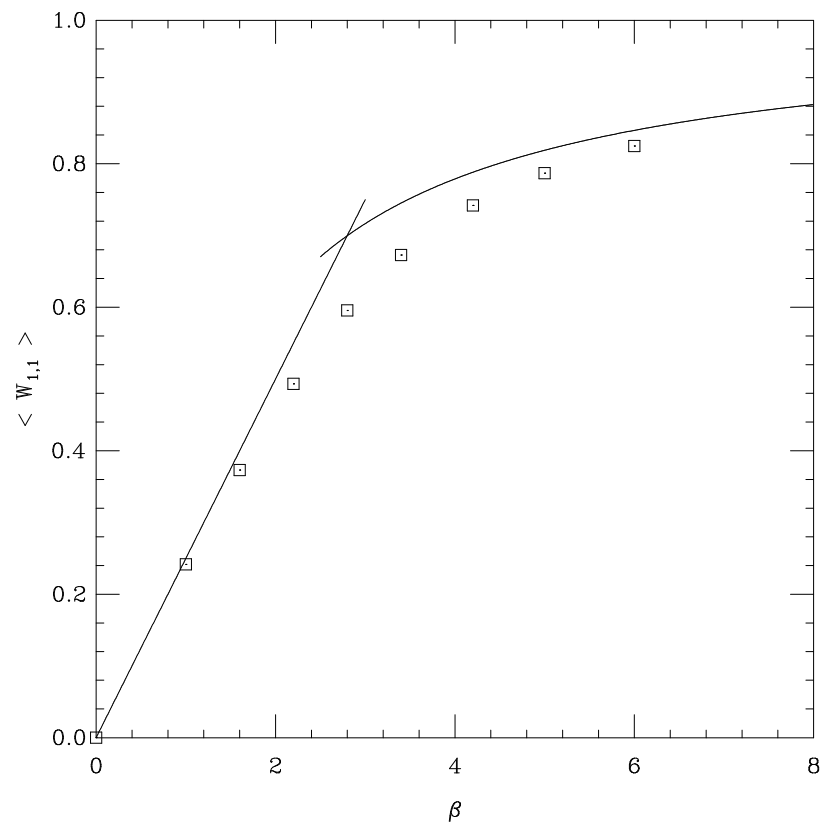

FIG. 1. Plot of the average plaquette $\left\langle W_{1,1}\right\rangle$ as a function of the coupling $\beta$. For comparison we also plot the leading strong-coupling expansion $\beta / 4$ and weak-coupling expansion $\exp (-1 / \beta)$. Error bars are not visible.

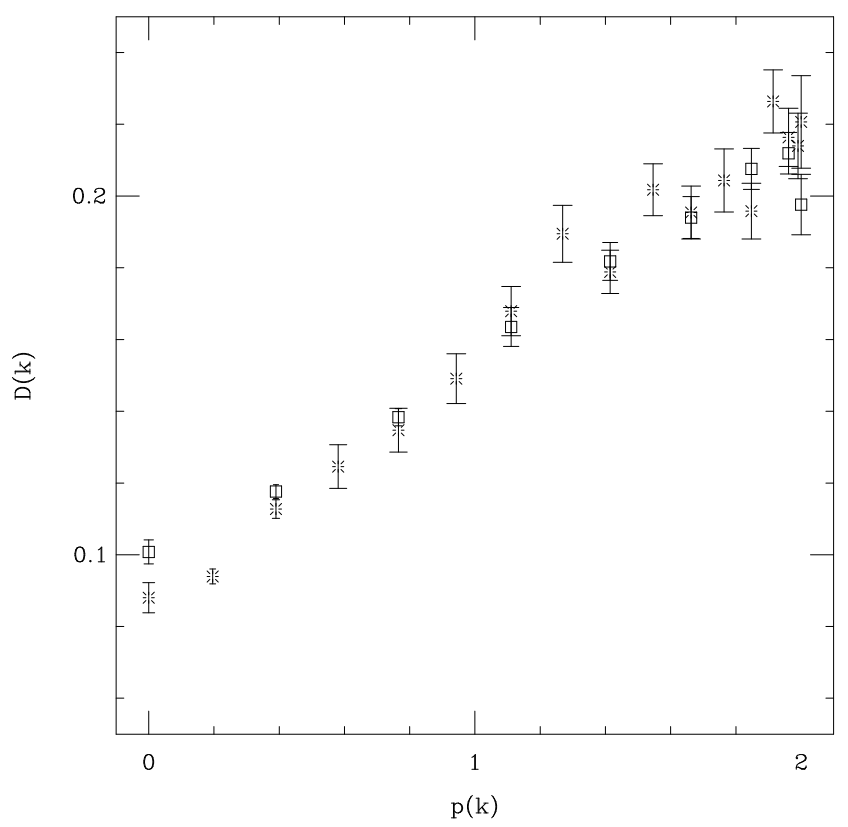

FIG. 2. Plot of the lattice gluon propagator $D(k)$ [see eqs. (8) and (9)] as a function of the lattice momentum $p(k)$ [see eq. (10)] for lattice volumes $V=16^{3}(\square)$ and $V=32^{3}(*)$, with $k=\left(0,0, k_{t}\right)$, at $\beta=0$. Error bars are one standard deviation. 

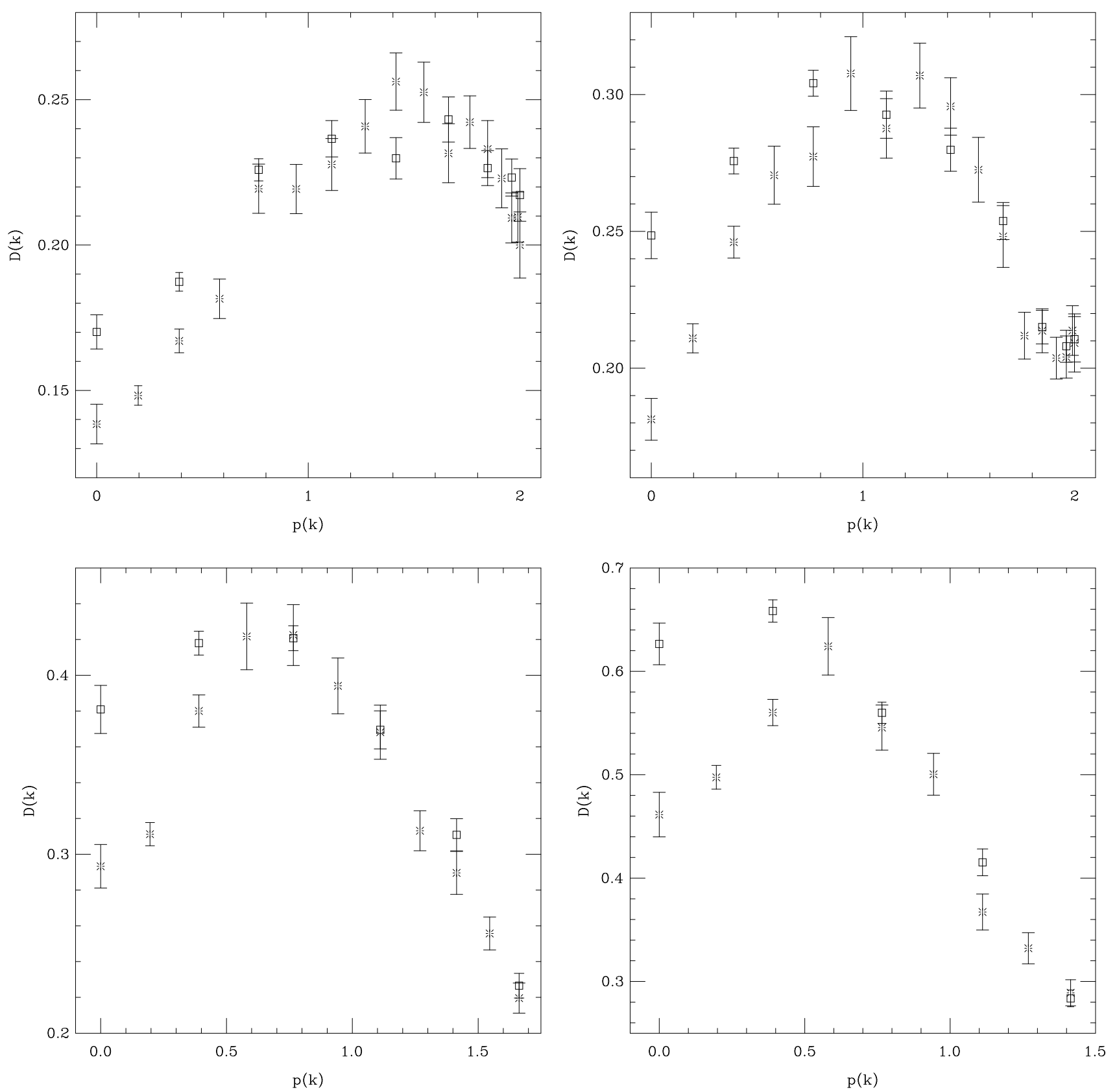

FIG. 3. Plot of the lattice gluon propagator $D(k)$ [see eqs. (8) and (9)] as a function of the lattice momentum $p(k)$ [see eq. (10)] for lattice volumes $V=16^{3}(\square)$ and $V=32^{3}(*)$, with $k=\left(0,0, k_{t}\right)$, at: (a) $\beta=1.0 ;(\mathbf{b}) \beta=1.6 ;(\mathbf{c}) \beta=2.2 ;(\mathbf{d})$ $\beta=2.8$. Error bars are one standard deviation. 

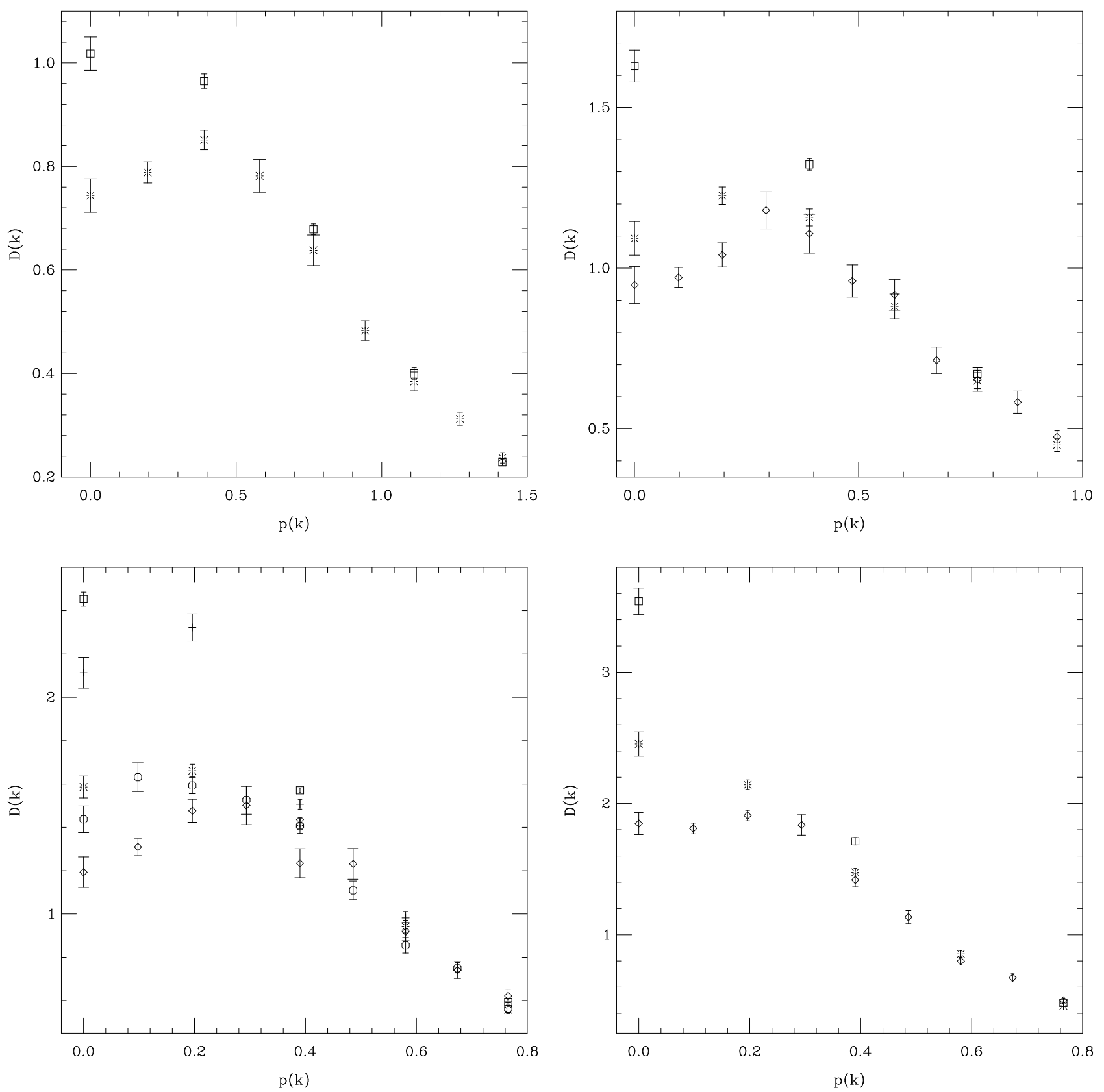

FIG. 4. Plot of the lattice gluon propagator $D(k)$ [see eqs. (8) and (9)] as a function of the lattice momentum $p(k)$ [see eq. (10)] for lattice volumes $V=16^{3}(\square), V=16^{2} \times 32(+), V=32^{3}(*), V=32^{2} \times 64(\circ)$, and $V=64^{3}(\diamond)$, with $k=\left(0,0, k_{t}\right)$, at: (a) $\beta=3.4$; (b) $\beta=4.2$; (c) $\beta=5.0 ;$ (d) $\beta=6.0$. Error bars are one standard deviation. 

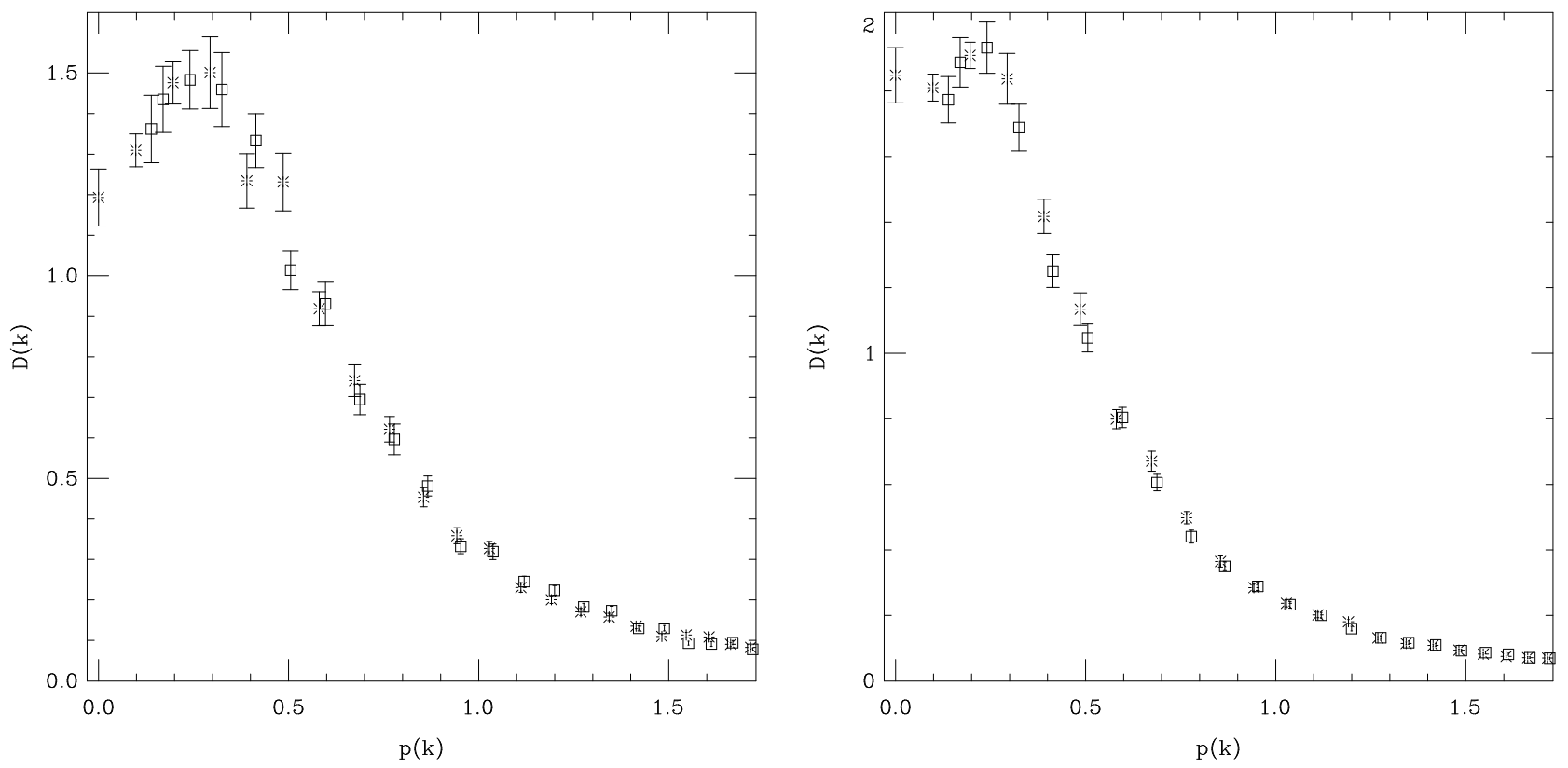

FIG. 5. Plot of the lattice gluon propagator $D(k)$ [see eqs. (8) and (9)] as a function of the lattice momentum $p(k)$ [see eq. (10)] for lattice volume $V=64^{3}$, with $k=\left(0,0, k_{t}\right)(*)$, and $k=\left(1,1, k_{t}\right)(\square)$, at: (a) $\beta=5.0$; (b) $\beta=6.0$. Error bars are one standard deviation.
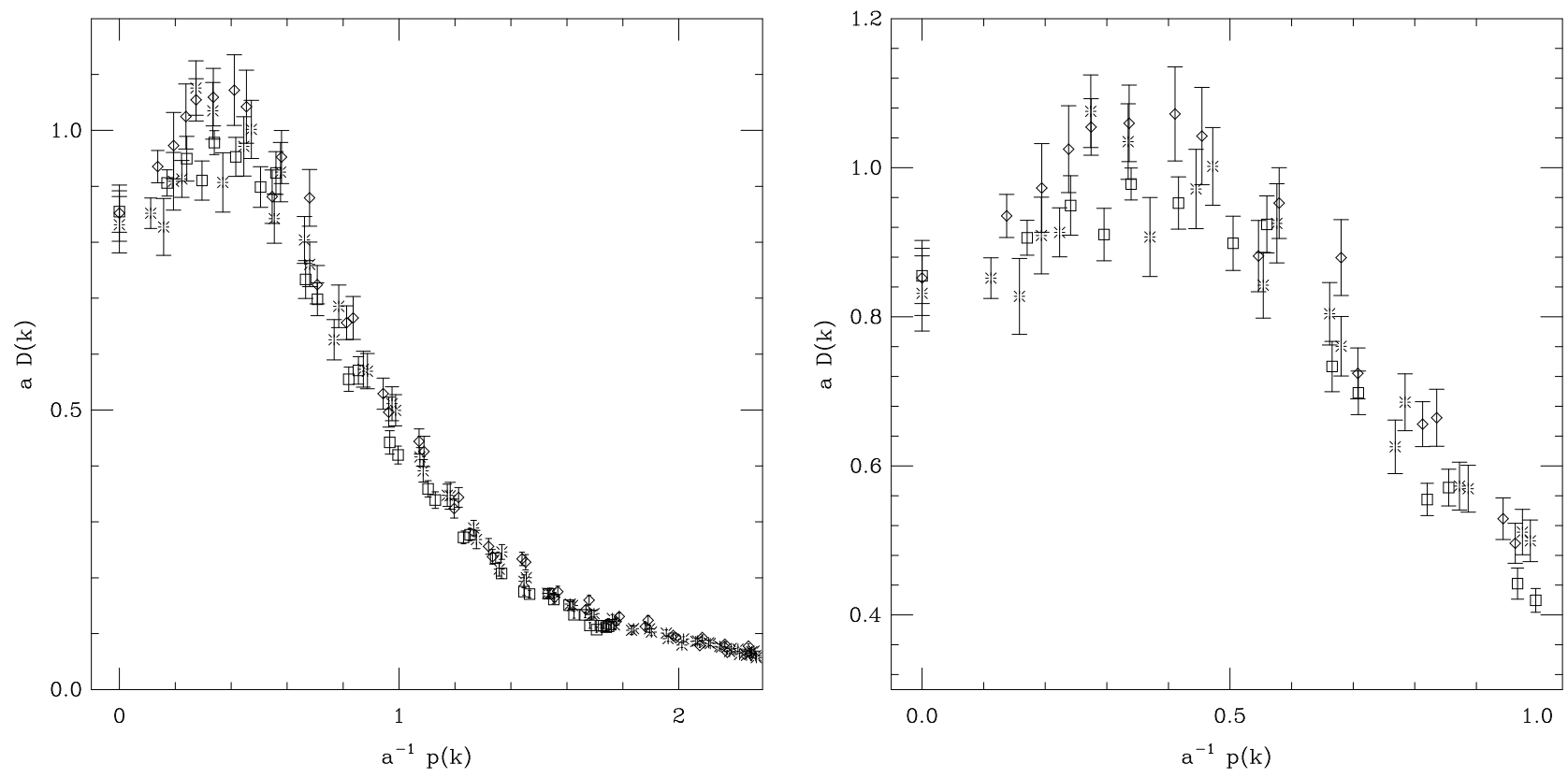

FIG. 6. Plot of the gluon propagator $a D(k)$ in physical units $\left(\mathrm{GeV}^{-1}\right)$ as a function of the momenta $a^{-1} p(k)$ (in $\mathrm{GeV}$ ) with $k=\left(0,0, k_{t}\right)$ and $k=\left(1,1, k_{t}\right)$, for $\beta=3.4$ and $V=32^{3}(\square), \beta=4.2$ and $V=64^{3}(*), \beta=5.0$ and $V=64^{3}(\diamond)$. In the second figure only the infrared region $a^{-1} p(k) \lesssim 1 \mathrm{GeV}$ is considered. Error bars are one standard deviation. 

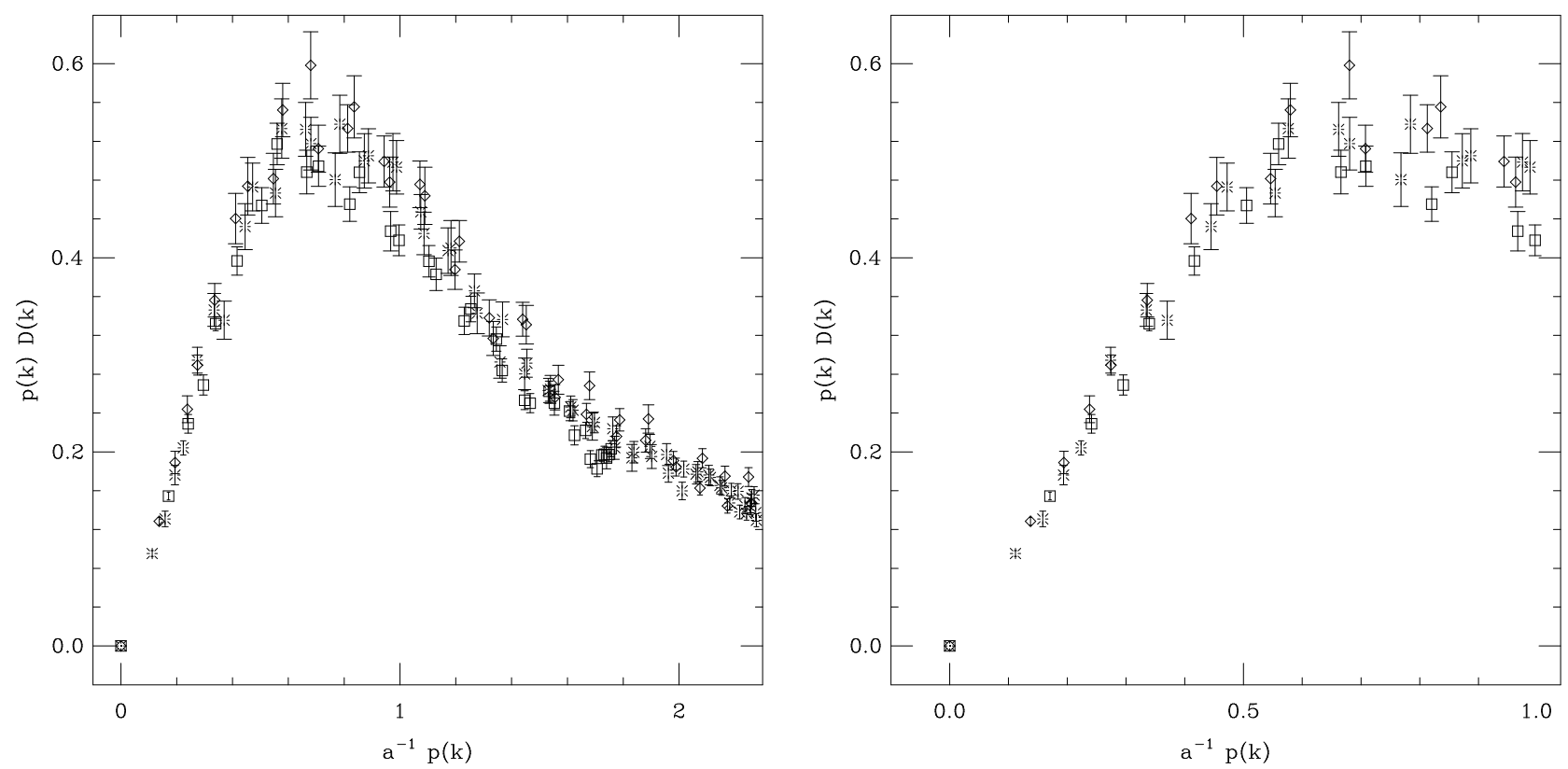

FIG. 7. Plot of the dimensionless product $p(k) D(k)$ as a function of the momenta $a^{-1} p(k)$ (in $\left.\mathrm{GeV}\right)$ with $k=\left(0,0, k_{t}\right)$ and $k=\left(1,1, k_{t}\right)$, for $\beta=3.4$ and $V=32^{3}(\square), \beta=4.2$ and $V=64^{3}(*), \beta=5.0$ and $V=64^{3}(\diamond)$. In the second figure only the infrared region $a^{-1} p(k) \lesssim 1 \mathrm{GeV}$ is considered. Error bars are one standard deviation. 
TABLE I. The pairs $(\beta, V)$ used for the simulations, the number of configurations, the number of HOR sweeps used for thermalization, the number of HOR sweeps between two consecutive configurations used for evaluating the gluon propagator, and the parameter $p_{s o}$ used by the stochastic overrelaxation algorithm.

\begin{tabular}{|c|c|c|c|c|c|}
\hline$\beta$ & $V$ & Configurations & Thermalization & Sweeps & $p_{\text {so }}$ \\
\hline$\overline{0.0}$ & $16^{3}$ & 200 & 4 & 2 & 0.85 \\
\hline 0.0 & $32^{3}$ & 100 & 4 & 2 & 0.90 \\
\hline$\overline{1.0}$ & $16^{3}$ & 200 & 1100 & 100 & $\overline{0.84}$ \\
\hline$\overline{1.6}$ & $16^{3}$ & 200 & 1650 & 150 & $\overline{0.81}$ \\
\hline 1.6 & $32^{3}$ & 100 & 1650 & 150 & 0.83 \\
\hline$\overline{2.8}$ & $16^{3}$ & 200 & 2750 & 250 & $\overline{0.72}$ \\
\hline 2.8 & $32^{3}$ & 100 & 2750 & 250 & 0.75 \\
\hline 3.4 & $16^{3}$ & 200 & 3025 & 275 & 0.69 \\
\hline 3.4 & $32^{3}$ & 100 & 3025 & 275 & 0.72 \\
\hline$\overline{4.2}$ & $16^{3}$ & 200 & 3300 & 300 & $\overline{0.66}$ \\
\hline 4.2 & $32^{3}$ & 100 & 3300 & 300 & 0.70 \\
\hline 5.0 & $32^{3}$ & 170 & 3325 & 325 & 0.68 \\
\hline 5.0 & $32^{2} \times 64$ & 100 & 3575 & 325 & 0.65 \\
\hline 5.0 & $64^{3}$ & 54 & 2275 & 325 & 0.69 \\
\hline$\overline{6.0}$ & $16^{3}$ & 200 & 3850 & 350 & 0.61 \\
\hline 6.0 & $32^{3}$ & 150 & 3350 & 350 & 0.67 \\
\hline 6.0 & $64^{3}$ & 97 & 3850 & 350 & 0.71 \\
\hline
\end{tabular}

TABLE II. For each coupling $\beta$ we report the value of the average plaquette $\left\langle W_{1,1}\right\rangle$, together with the volume $V$ and the number of HOR sweeps used for the analysis. Also, for $\beta \geq 3.4$, we report the tadpole-improved coupling $\beta_{I}$, the string tension $\sqrt{\sigma}$ in lattice units, and the inverse lattice spacing $a^{-1}$ in $\mathrm{GeV}$. Error bars for the string tension and the inverse lattice spacing come from propagation of errors. Error bars for $\left\langle W_{1,1}\right\rangle$ are one standard deviation, evaluated taking into account the value of the integrated autocorrelation time $\tau_{i n t, W_{1,1}}$ for the plaquette, namely the variance is multiplied by $2 \tau_{i n t, W_{1,1}}$ (see eq. $(3.7)$ in Reference 29]).

\begin{tabular}{cccccc}
\hline \hline$\beta$ & $V$ & Sweeps & $\left\langle W_{1,1}\right\rangle$ & $\beta_{I}$ & $\sqrt{\sigma}$ \\
\hline 0.0 & $32^{3}$ & 198 & $0.000110(113)$ & & \\
1.0 & $32^{3}$ & 9900 & $0.241650(16)$ & & \\
1.6 & $32^{3}$ & 14850 & $0.373147(13)$ & & \\
2.2 & $32^{3}$ & 19000 & $0.493302(12)$ & & \\
2.8 & $32^{3}$ & 24500 & $0.595483(11)$ & $2.28725(3)$ & $0.506(13)$ \\
3.4 & $32^{3}$ & 26950 & $0.672720(9)$ & $3.11582(2)$ & $0.387(8)$ \\
4.2 & $64^{3}$ & 14149 & $0.741862(4)$ & $3.93438(1)$ & $0.314(5)$ \\
5.0 & $64^{3}$ & 16164 & $0.786877(3)$ & $4.94870(2)$ & $0.254(4)$ \\
6.0 & $64^{3}$ & 25135 & $0.824783(3)$ & $1.14(2)$ & $1.40(2)$ \\
\hline \hline
\end{tabular}

TABLE III. For each coupling $\beta \geq 3.4$ we report the lattice spacing in fm, the largest lattice volume $V_{\text {max }}$, the corresponding physical volume in $\mathrm{fm}^{3}$, and the smallest non-zero momentum that can be considered for that lattice (in GeV).

\begin{tabular}{lcccc}
\hline \hline$\beta$ & $a(\mathrm{fm})$ & $V_{\max }$ & $a^{3} V_{\max }\left(\mathrm{fm}^{3}\right)$ & $a^{-1} p_{\min }(\mathrm{GeV})$ \\
\hline 3.4 & $0.226(6)$ & $32^{3}$ & $7.2^{3}$ & 0.171 \\
4.2 & $0.173(3)$ & $64^{3}$ & $11.1^{3}$ & 0.112 \\
5.0 & $0.140(2)$ & $64^{3}$ & $9.0^{3}$ & 0.137 \\
6.0 & $0.114(1)$ & $64^{3}$ & $7.30^{3}$ & 0.170 \\
\hline \hline
\end{tabular}

